\title{
Stress analysis of an agitated particle bed with different particle aspect ratios by the discrete element method
}

\author{
Wei Pin Goh ${ }^{1}$, Mojtaba Ghadiri ${ }^{1, *}$, Frans Muller ${ }^{1}$, Kushal Sinha ${ }^{2}$, Nandkishor Nere $^{2}$, Raimundo $\mathrm{Ho}^{2}$, Shailendra \\ Bordawekar $^{2}$ and Ahmad Sheikh ${ }^{2}$ \\ ${ }^{1}$ School of Chemical and Process Engineering, University of Leeds, Leeds, UK \\ ${ }^{2}$ Process Research and Development, AbbVie Inc., 1 North Waukegan Road, North Chicago, Illinois 60064, USA
}

\begin{abstract}
The size distribution, shape and aspect ratio of particles are the common factors that affect their packing in a particle bed. Agitated powder beds are commonly used in the process industry for various applications. The stresses arising as a result of shearing the bed could result in undesirable particle breakage with adverse impact on manufacturability. We report on our work on analysing the stress distribution within an agitated particle bed with several particle aspect ratios by the Discrete Element Method (DEM). Rounded cylinders with different aspect ratios are generated and incorporated into the DEM simulation. The void fraction of the packing of the static and agitated beds with different particle aspect ratios is analysed. Principal and deviatoric stresses are quantified in the regions of interest along the agitating impeller blade for different cases of particle aspect ratios. The relationship between the particle aspect ratio and the stress distribution of the bed over the regions of interest is then established and will be presented.
\end{abstract}

\section{Introduction}

Agitated powder beds are found in many process industries for mixing and drying. The process performance is affected by the physical properties of the particles such as size and shape [1,2]. Different packings of the bed give rise to different extents of resistance to flow and this may have adverse effects on particle breakage and transformations [3]. DEM is used to study the stress distribution within the region of interest as a function of particle aspect ratio. Rounded cylinders are used to represent particles with different aspect ratios. Polyhedra geometry [4] is used to make the particles to simulate particle shape. When a particle bed is agitated, the stresses arising within the bed change over time because of the impeller agitation. The interactions between the particles and the impeller as well as the evolution of stresses are of particular interest in this study. The bed packing void fraction is analysed and the stresses are characterised for various particle aspect ratios.

\section{Materials and Methods}

\subsection{Simulation Setup}

DEM simulations were carried out using the ROCKY software (ESSS, Florianópolis, Brazil) by incorporating particle shape as polyhedra. Particles with aspect ratios in the range $1,1.25,1.5,1.75,2,3$ and 5 were investigated. As can be seen in Figure 1, spheres are used to represent particles with unity aspect ratio while particles with other aspect ratios are represented by rounded cylinders with different lengths and diameters. The volume of the particles is kept constant at an equivalent-volume diameter of $3 \mathrm{~mm}$ regardless of the change of particle aspect ratio throughout the simulations. The geometry of the impeller and vessel used in the simulation are reproduced as shown in Figure 2. The details of the geometry and simulation conditions are shown in Table 1. The vessel is initially fed with particles until the bed height $(0.06 \mathrm{~m})$ is approximately three times the blade height $(0.018 \mathrm{~m})$. The bed is then agitated at a speed of 1 $\mathrm{rev} / \mathrm{s}$ for $5 \mathrm{~s}$.

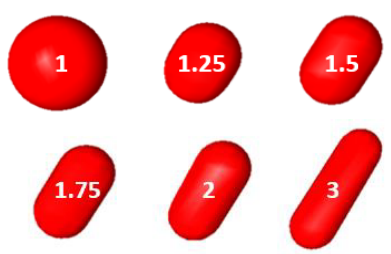

Figure 1. Sphere and rounded cylinders with different aspect ratios.
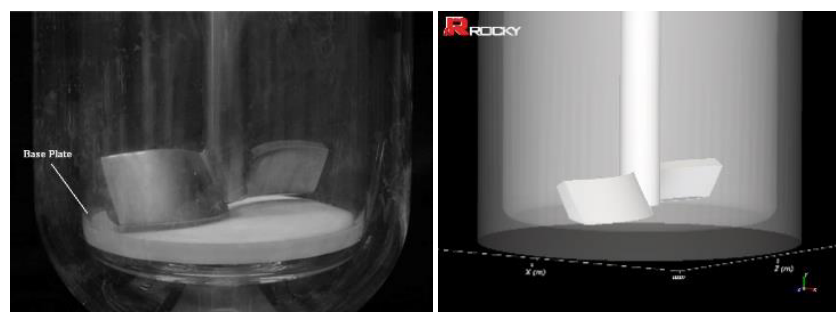

Figure 2. Actual impeller and vessel (left) and its CAD representation (right).

\footnotetext{
${ }^{*}$ Corresponding author: $\underline{\text { m.ghadiri@,leeds.ac.uk }}$
} 
Table 1. Geometry and simulation details.

$\begin{array}{lc}\text { Operating Conditions } & \\ \text { Vessel Inner Diameter (mm) } & 110 \\ \text { Number of Blades (-) } & 2 \\ \text { Blade Height (mm) } & 18 \\ \text { Blade Inclination }\left({ }^{\circ}\right) & 60 \\ \text { Blade Diameter }(\mathrm{mm}) & 98 \\ \text { Clearance Height }(\mathrm{mm}) & 2 \\ \text { Angular Velocity }(\mathrm{rev} / \mathrm{s}) & 1 \\ \text { Particle Feed Rate }(\mathrm{kg} / \mathrm{s}) & 3 \\ \text { Particle Density }\left(\mathrm{kg} / \mathrm{m}^{3}\right) & 1.6 \\ \text { Young's Modulus }(\mathrm{Pa}) & 10^{7} \\ \text { Coefficient of Sliding Friction }(-) & 0.3 \\ \text { Coefficient of Restitution }(-) & 0.3 \\ \text { Simulation Time (s) } & 5\end{array}$

\subsection{Particle Bed Void Fraction}

Particles with different aspect ratios pack differently to form a bed and this in turn affects the prevailing shear stress[5]. In this study, a cylindrical measurement cell with a height of $0.02 \mathrm{~m}$ is generated to measure the void fraction of the particle bed (Figure 3). The measurement cylinder is placed right above the impeller blades and below the free surface of the bed to eliminate the situation where unwanted entities (such as impeller blades and area above free surface of the bed) are taken into the calculation of the void fraction.

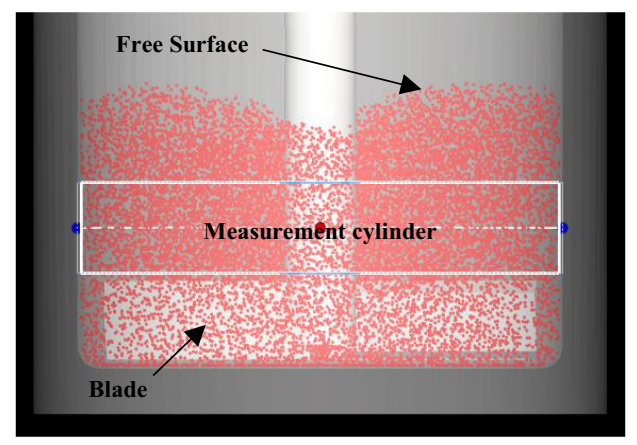

Figure 3. Position of measurement cylinder.

\subsection{Stress Analysis}

In an agitated bed, as the impeller rotates, particles in front of the blades experience shear and get pushed over and fall behind the blade to a region where the bed is dilated. The prevailing stresses of the region of particle bed that comes into direct contact with the rotating bed are analysed. Experimentally, the stress distribution within a particle bed is too difficult to be determined, but this is made possible in DEM simulation by introducing measurement bins in the regions of interest [6]. Five measurement bins created along the impeller blade that capture the particle interactions during each time step within their own domain are shown in Figure 4. The stress tensor of each bin is calculated using the equation below developed by Bagi [7],

$$
\sigma_{i i}=\frac{1}{V} \sum_{1}^{N} F_{i i} \cdot r_{p}
$$

where $\sigma$ is the stress, $V$ is the measurement bin volume, $N$ is the number of particle within the bin and $F$ is the force acting on a particle in direction $i i$ with radius $r_{p}$. The average principal and deviatoric stresses are then given by equations 2 and 3 :

$$
\sigma_{\text {ave }}=\frac{P_{1}+P_{2}+P_{3}}{3}
$$

where $P_{1}, P_{2}$ and $P_{3}$ are the three principal stresses, respectively. The deviatoric stress, $\sigma_{d e v}$ [8] can be calculated from the principal stresses and is defined as

$$
\sigma_{d e v}=\frac{\sqrt{\left(P_{1}-P_{2}\right)^{2}+\left(P_{1}-P_{3}\right)^{2}+\left(P_{2}-P_{3}\right)^{2}}}{\sqrt{6}}
$$

The calculation for both principal and deviatoric stresses is performed at each time step to capture the stresses experienced along the impeller blade. The size of the bin is kept in such a way that it has approximately more than 100 particles in each cell at each time step so that the dependency of individual particles is minimised while improving the statistical reliability for averaging the stress tensor in a measurement bin.

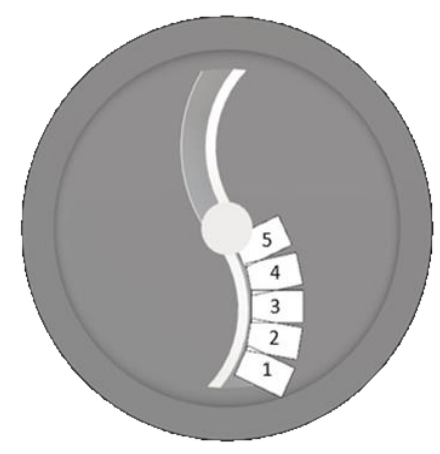

Figure 4. Measurement bins along the impeller blade.

\section{Results and Discussions}

\subsection{Bed Packing}

The void fraction of the static and agitated beds for different particle aspect ratios is shown in Figure 5. A bed made with spherical particles (aspect ratio 1) appears to have a void fraction of 0.5 during static state. As the particle aspect ratio increases, the void fraction of the bed decreases initially. A minimum void fraction value is observed when the particle aspect ratio is 1.5. A further increase in the particle aspect ratio increases the void fraction. While the bed is agitated, higher void fractions of the particle bed are observed, suggesting that the bed is 
dilated when it is in motion. The arrangement of particles at the middle of the bed (cross sectional view at XY plane) when it is static $(0 s)$ and agitated $(5 s)$ is shown in Figure 6. At low aspect ratio from 1 to 1.75 , the particle arrangement appears random for both static and dynamic cases. As the particle aspect ratio approaches 2, the tendency of the particles to reorientate themselves and align with the neighbouring particles becomes prevalent. As the particle aspect ratio increases, the number of particles that cluster together increases as well. The effect is most significant and can be seen clearly for the case of particles with aspect ratio of 5 . Even when the bed is agitated, the phenomenon of particles clustering as groups still prevails.

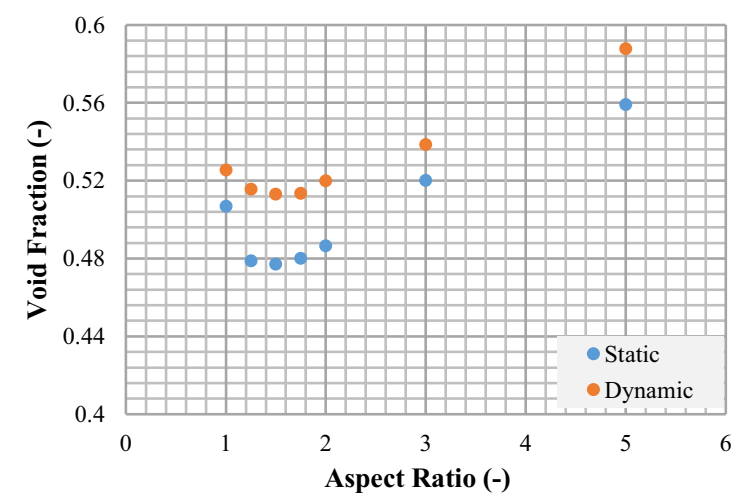

Figure 5. Void fraction for particle beds with different particle aspect ratios.

\subsection{Impeller Torque}

The impeller torque data obtained for the mono-sized particles are shown in Figure 7. A high torque is observed at the initial period when the impeller starts rotating. This is due a number of reasons: (i) rapid acceleration of the impeller that immediately achieves the required velocity, (ii) particle bed dilation and (iii) ordering of crystallinelike structure that occurs when the particles are monosized. As the particle bed starts to move, the torque reduces and begins to plateau. Increasing the particle aspect ratio increases the impeller torque. However, for particle aspect ratios more than 1.5 , the torque is independent of the aspect ratio. A plot of the average impeller torque versus the aspect ratio shows a better visualisation of the trend (Figure 8). The impeller torque increases initially and starts to stabilise at a particle aspect ratio of around 2. A further increase in the particle aspect ratio only increases the impeller torque marginally, suggesting that there is a limiting particle aspect ratio where further any increase yields no significant effect on the impeller torque. Visual observation from Figure 6 suggested that the particles tend to align themselves and form groups of clusters that have preferred orientation when the aspect ratio is more than 1.75 , which could potentially explain the trend observed. However, higher fluctuations of the impeller torque are observed as the particle aspect ratio increases and this could be attributed to the fact that particles with higher aspect ratio have a higher tendency of jamming.

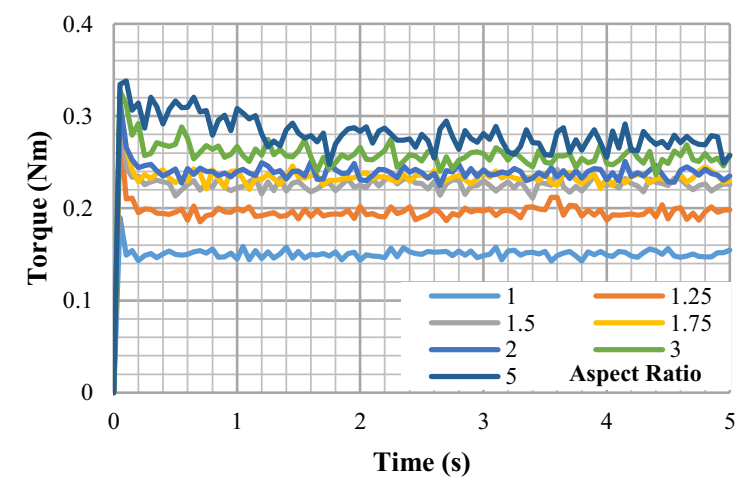

Figure 7. Impeller torque as a function of time for particle beds with different particle aspect ratios.

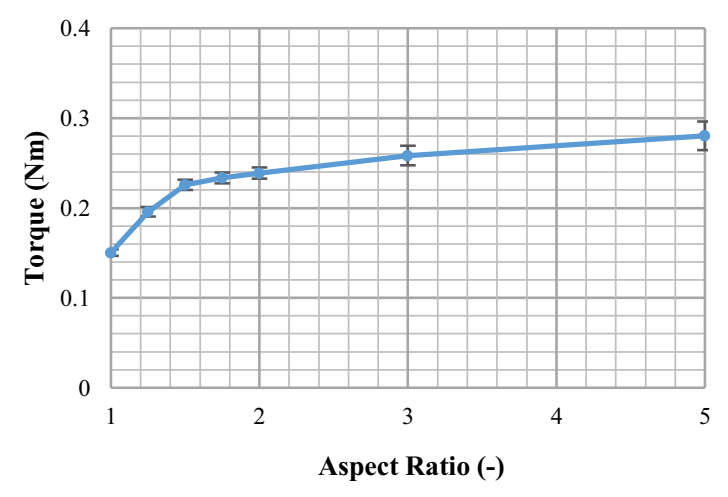

Figure 8. Average impeller torque versus particle aspect ratio.

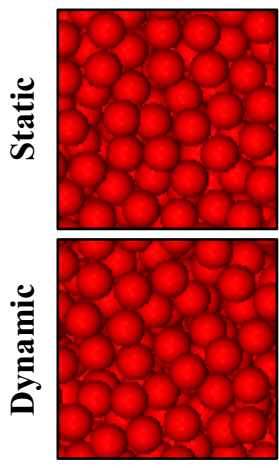

1
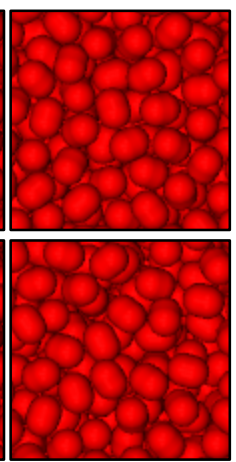

1.25
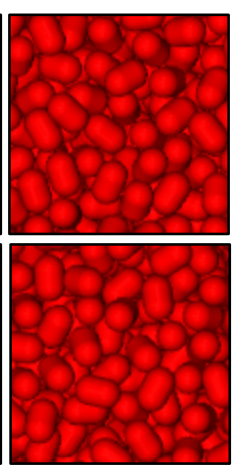

1.5
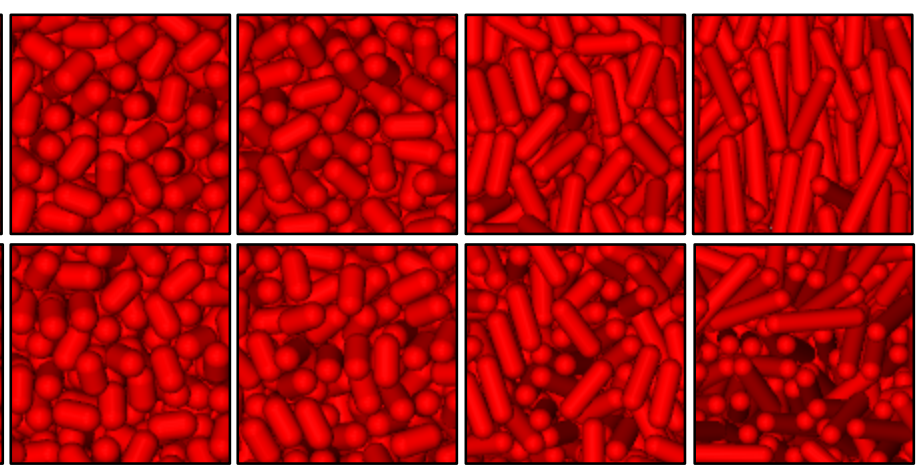

1.75

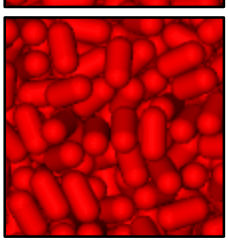

2

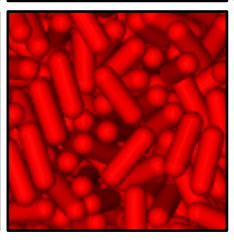

5

Figure 6. Particle arrangement in static and dynamic beds (cross sectional view at XY plane). 


\subsection{Principal and Deviatoric Stresses}

When the impeller is rotating, for every $0.5 \mathrm{~s}$, a blade comes into direct contact with the measurement bins. The region in front of the blade is constantly being compressed, sheared and pushed over the blade to a region behind it where the particle bed is relaxed. The deviatoric stresses experienced at the region nearest to the wall (bin 1) over time are shown in Figure 9. As the blade approaches the bins, an increase in the deviatoric stresses is observed. Upon reaching a maximum value, the deviatoric stresses decrease as the blade moves past the measurement bins. This cyclical event is observed for every measurement bin for both principal and deviatoric stresses but the results are not shown here. The average principal and deviatoric stresses experienced at the regions near the vessel wall (bin 1) and near the impeller shaft (bin 5) are shown in Figures 10 and 11. The principal stresses increase with the increase of particle aspect ratio for both the regions near the vessel wall and near the impeller shaft but the rate of increase is different. Similar trend is observed for deviatoric stresses. Compared to bin 5 , a substantial increase of stresses (6 times) is observed at bin 1 as the particle aspect ratio is increased. This suggests that the particles are subjected to different extents of stresses at different radial positions. Both principal and deviatoric stresses show fluctuations as the particle aspect ratio is increased.

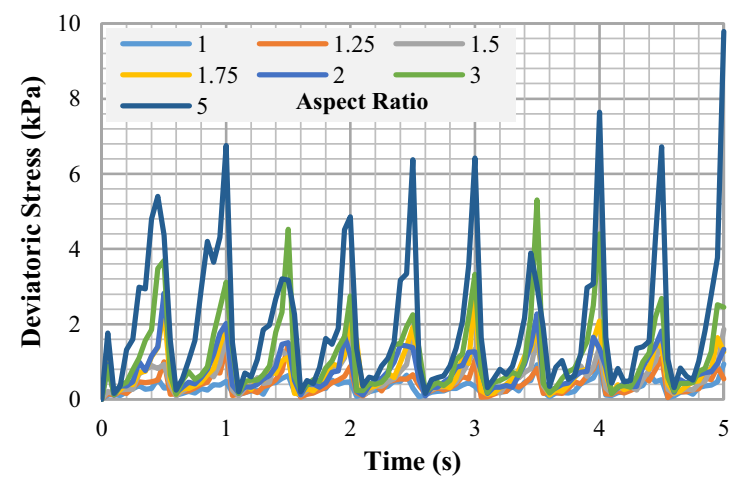

Figure 9. Deviatoric stress experienced at the region near the wall over time.

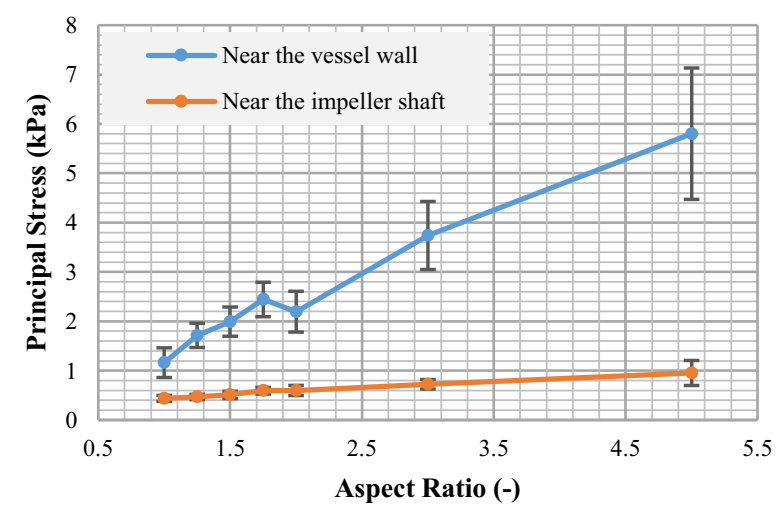

Figure 10. Average principal stress at the regions near the shaft and near the vessel wall.

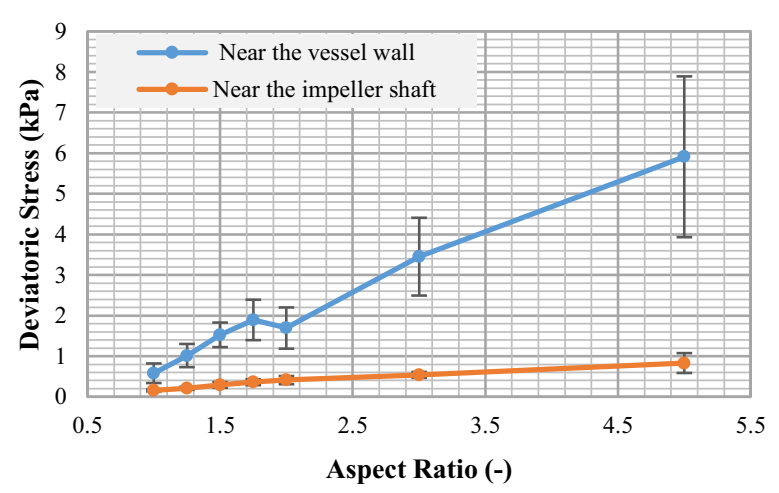

Figure 11. Average deviatoric stress at the regions near the shaft and near the vessel wall.

\section{Conclusions}

The packing of particle beds under shear deformation by an impeller is assessed using DEM for different particle aspect ratios of rounded cylinders from 1 to 5 and also for spherical particles for comparison. The void fraction of both static and agitated beds is calculated as compared. The beds undergo compression and dilation when being agitated. The impeller torque which is a measure of the resistance of the bed to flow is found to increase with the particle aspect ratio initially and it then reaches a plateau. Both principal and deviatoric stresses are evaluated at the regions of interest and are found to increase along the radial position.

\section{Acknowledgment}

The financial support of AbbVie Inc., Chicago, USA, for a studentship for the first author is gratefully acknowledged. Kushal Sinha, Nandkishor Nere, Raimundo Ho, Shailendra Bordawekar and Ahmad Sheikh are present employees of AbbVie Inc.

\section{References}

1. G. Cho, J. Dodds, and J. C. Santamarina, J. Geotech. Geoenviron. Eng., 132, 591-602 (2006)

2. R. Guises, J. Xiang, and J. L. A. Munjiza, Granul Matter, 11, 281-292 (2009)

3. A. Potapov and C. Campbell, Int. J. Mod. Phys. C., 7, 717-729 (1996)

4. C. Hare, M. Ghadiri, and R. Dennehy, Chem. Eng. Sci., 66, 4757-4770 (2011)

5. Y. Guo, C. Wassgren, W. Ketterhagen, B. Hancock, and B. James, J. Fluid Mech, 713, 1-26 (2012)

6. K. Bagi, Mech. Mater., 22, 165-177 (1996)

7. S. Luding, Particuology, 6, 501-505 (2008) 